\title{
A double-motor structure energy-saving Ac speed regulating system
}

\author{
Liu juan $^{1, a}$, Jin Laizhuan ${ }^{2}$, Lan yaxun $^{1}$ \\ (1. Guangzhou vocational college of science and technology, Guangzhou, 510550, China) \\ (2. Information Engineering College, Taiyuan University of Technology, Taiyuan 030024,China) \\ ${ }^{a}$ E-mail: $\underline{\text { czsem@163.com }}$
}

Key words:STM32; frequency difference control; AC motor; SVPWM;

Abstract: This paper introduces the structure of the double AC motors speed-regulating experimental system and control methods of the system, and describes the application of the STM32 microcontroller hardware module design and programming. With the SVPWM technology, software flow chart based on STM32 MCU are also given. Compared with traditional speed control system, the system has high efficiency and energy saving, low-cost and flexible superior speed performances.

\section{Double-motor structure of AC speed control experiment system}

This experimental system based on STM32 microcontroller controlled is shown in Fig.1. Compared with traditional speed control system, the system architecture has been significant improved. It uses an AC motor as dummy load and changes the structure of conventional coaxial motor-generator group into the structure of coaxial motor-motor group, and the drive power is composed of two parallel inverters and frequency difference control circuit. The output voltages from two parallel inverters, respectively drive two motors. From the energy point of view the system has the following advantages:

(1)For two inverters DC bus side using parallel mode, the voltages are equal, thus both of inverters public a rectifier bridge, reducing the switching losses;

(2)Energy of generator regeneration is completely absorbed by motor through the DC bus, thereby reducing the electrical energy from the power grid. It fully embodies the energy-saving ideas. Therefore, the system will not only be able to complete the AC motor speed control experiment, but also has good energy saving features and high performances. 


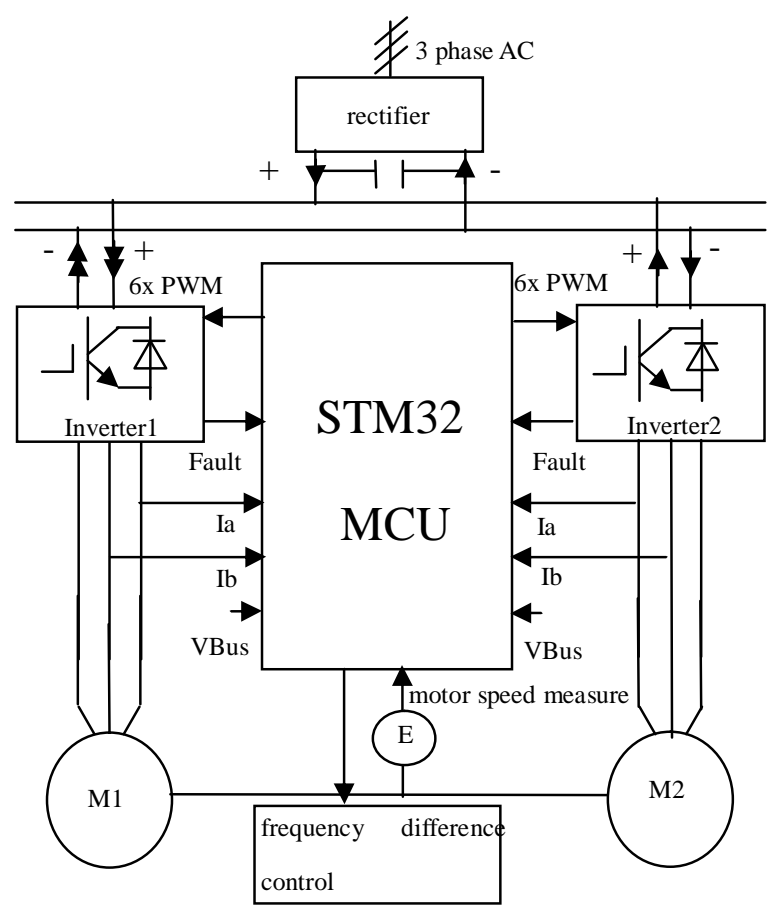

Fig .1 AC variable speed dual-motor experimental system architecture diagram

\section{The principle of frequency difference}

Frequency difference control is a method that changes the frequency difference between the two motors to control the electrical load size and the operational status. In Fig.1, if two control frequencies of motors M1 and $\mathrm{M} 2$ are respectively $\mathrm{f}_{1}, \mathrm{f}_{2}$ and not equal, so that there is frequency difference $\Delta \mathrm{f}$, thus according to the speed of induction motor formula:

$$
\mathrm{n}=60 \mathrm{f}(1-\mathrm{s}) / \mathrm{P}_{\mathrm{n}} \quad \mathrm{n}_{0}=60 \mathrm{f} / \mathrm{P}_{\mathrm{n}}
$$

If $\mathrm{f} 1 \neq \mathrm{f}_{2}\left(\right.$ set $\left.\mathrm{f}_{1}>\mathrm{f}_{2}\right)$, then $\mathrm{n}_{01}>\mathrm{n}_{02}$, it is said that the two no-load speeds of motors are not equal. As the two-motor coaxial connection, so when steady-state the two motors torque and speed are equal as Fig. 2 shown below.

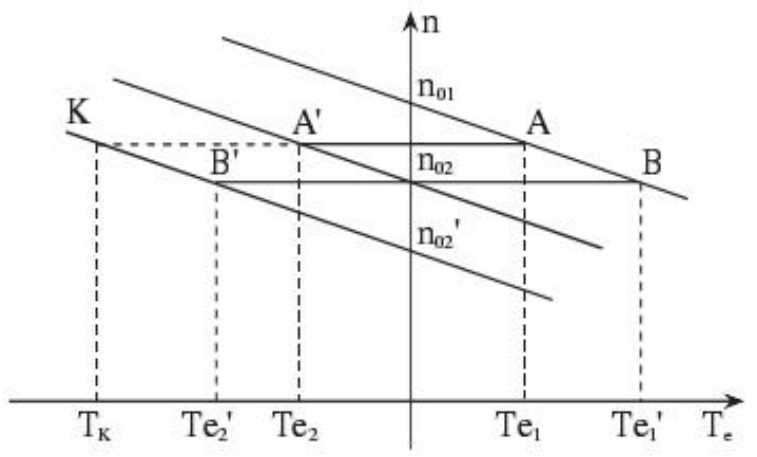

Fig. 2 Mechanical features of two motors

M1 runs at motor state (A point), meanwhile $\mathrm{M} 2$ runs at power generation state (A' points). The magnetic torque of M2 is the load torque of M1. If ruducing the control frequency $f_{2}$ while the control frequency $f_{1}$ is unchanged, so the frequency difference is increases, then the no-load speed of M2 is reduced to $\mathrm{n}_{02}$. As the rotor speed can not sudden changes, so that $M 2$ switches to operating point $K\left(T_{k}, n_{1}\right)$ of $n_{02}$ corresponding to the mechanical characteristic curve. At this time, $\mathrm{T}_{\mathrm{e} 1}<\mathrm{T}_{\mathrm{k}}$, namely, the magnetic torque of $\mathrm{M} 1$ is less than the load torque. From Fig. 2, we can see, this time M1 and M2 speed will drop, until it reaches a new equilibrium point $\mathrm{B}, \mathrm{B}^{\prime}$ point, at this time $\mathrm{T}_{\mathrm{e} 1}{ }^{\prime}=\mathrm{T}_{\mathrm{e} 2}{ }^{\prime}, \mathrm{n} 1^{\prime}=\mathrm{n} 2{ }^{\prime}$. Compared with the original equilibrium point, 
$\mathrm{T}_{\mathrm{e} 1}{ }^{\prime}>\mathrm{T}_{\mathrm{el}}, \mathrm{T}_{\mathrm{e} 2}{ }^{\prime}>\mathrm{T}_{\mathrm{e} 2}$, namely M1 magnetic torque and load torque are increased.

Similarly, if increasing the control frequency $f_{2}$, while $f_{1}$ unchanged, namely reducing frequency difference, then the no-load torque of M2 will increase, while M1 magnetic torque and load torque are reduced. If setting $\mathrm{f}_{1}<\mathrm{f}_{2}, \mathrm{n}_{01}<\mathrm{n}_{02}$, then $\mathrm{M} 1$ runs at power generation state, while $\mathrm{M} 2$ runs at motor state. The magnetic torque of M1 is the load torque of M2. Similarly, the size of the load and the torque can be changed by changing the frequency difference. When $\mathrm{f}_{1}=\mathrm{f}_{2}$, the frequency difference $\Delta \mathrm{f}=0, \mathrm{M} 1, \mathrm{M} 2$ are all running at non-load state.Through above analysis we can see, the system as Fig.1 shown can not only adjust the size of the frequency difference $\Delta \mathrm{f}$ to control the size of the motor torque, but also through changing the positive and negative $\Delta \mathrm{f}$ adjust the drive system working state so as to conveniently realize the motor state conversion.If setting M1, M2 reverse rotation, we can achieve the four-quadrant motor operation. When the system is running, if you set the frequency difference $\Delta \mathrm{f} \neq 0$, then one of the two motors must run at the motor state, and other must run at the power generation state. As both of inverters public a not-controlled rectifier bridge, renewable energy can not be back to power Grid, but the reverse not-controlled three phase bridge composed of diodes of inverters bridge arm in the circuit has provided the feedback path for the exchange of energy. Namely, In circuit loop which the motor running at power generation state, the role of inverter has been converted into the role of rectifier. The reverse diodes bridge of parallel inverter has completed the function that renewable $\mathrm{AC}$ voltage from the motor running at the state of power generation rectifier into DC voltage acting on the common DC bus. At the moment the direction of current of the inverter DC side is the opposite of current direction at inverter state, as Fig.1 circuit shown. During this period the input power of inverter 1 comes from the sum power supplied by power source and inverter2, that is, renewable power was completely absorbed by motor through the DC bus, thereby reducing the energy absorbed from the power grid. From this we can see that the experimental system in this mode renewable energy from M2 running at the state of power generation has been full used.

\section{The implementation of control system based on STM32 MCU}

STM32 , latest addition into CM3 processor family, is ultra-high integration single-chip MCU, suited to many applications. It has powerful platform for highly flexible, and two advantages of memory and the number of pins with widly range of changes and high-performance applications in cost-sensitive applications from simple to complex.As an ideal platform to achieve vector control (or field-oriented FOC) algorithm , high-performance vector control algorithm is widely used in drive. They can be control torque and speed accurately and quickly, and ensure the optimum efficiency in transient operation. Even more valuable is that, whether controlling induction or synchronous motors, it can use the same framework. When you need to cope with a variety of motor types, this consistency will undoubtedly give developers a significant burden reduction. Finally, trying to lower the cost of the drive ,the rotor speed and position sensorless algorithms come in handy. STM32 microcontroller provides low-cost, low-power, high-performance motor control processing. The chip integrates a number of effective peripherals: such as high-level timer TIM1, ordinary timer TIMx, serial communication module SCI, analog-digital conversion module A/D, Controller Area Network module CAN2.0 and so on. These peripherals provide more convenient to achieve such experimental system designed in this paper.

STM32 also provides STM32 program libraries, and the document of C firmware libraries is optimized and used for both of the PMSM and AC induction brushless motor. These libraries include different methods of current detection systems, such as the three shunt resistors, etc. Independent current detection mechanism and advanced methodology, the demand for bus voltage is lower.The library also includes a different rotor position feedback encoder. Such as the tachometer, Hall sensors, no sensor (designed for PMSM motor). These libraries provides more convenient for user to develope and utilize.

The principle structure of double-motor AC speed control experiment system is shown in Fig.1. The main 
circuit equiped with AC-DC-AC voltage source frequency converter, is mainly composed of rectifier, inverter, IPM drive circuit. Its working principle is transform three-phase currents into direct currents through the non-controlled rectifier. Inverter (or IPM) will be transforming DC voltage filtered into a voltage and frequency adjustable three-phase AC power source provided to the motor. Compared with ordinary IGBT modules, IPM has high system performance and its reliability is further improved . Particularly, IPM module has integrated drive and protection circuit, the system's hardware circuit is simple, reliable, and self-protective. STM32 micro-controller is the direct control part of the motor running and the core of the whole control system. STM32 microcontroller has two advanced timers .They can respectively generate six road PWM signals, and these PWM signals are sent to IPM of two coaxial transmission asynchronous induction motor. And an motor-motor mode AC speed control system is constituted, realizing double inverters precise working by setting any difference frequency。 Thereby a reversible magnetic torque load relation of the two coaxial motor is established. STM32 MCU has a quadrature encoder interface, establishing one to one relationship between the rotor position and the time of quadrature encoder interface. In order to obtain high-dynamic targets, through the encoder the motor speed is reads and then feedback to the given input clients (converted to frequency value). The frequency is respectively compared with given frequencies $\mathrm{f}_{1}{ }^{*}, \mathrm{f}_{2} *$. So that a speed closed-loop FOC control is constituted. The Principle Structure of FOC drive in a speed loop based on dual-motor structrue is shown in Fig.3.

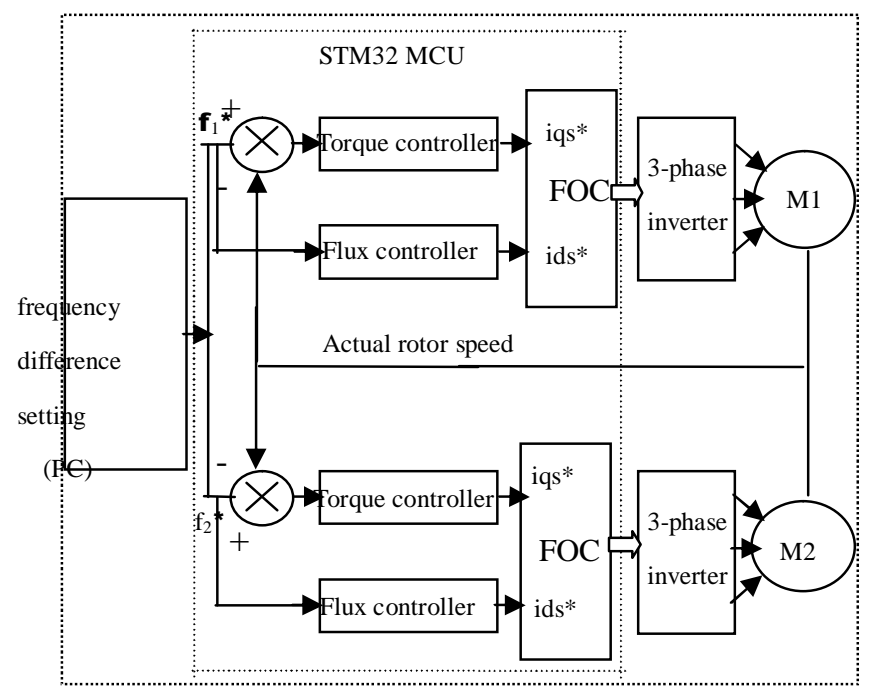

Fig.3 FOC drive in a speed loop of dual-motor structrue.

\subsection{High-precision flexible PWM output}

STM32 microcontroller's advanced timer driven by a programmable prescaler contains a 16-bit auto-reload counter. Clock frequency is twice the APB frequency ,reaching as high as $72 \mathrm{MHz}$, providing 13.8ns timing accuracy. Advanced timer can generate 6-channel programmable PWM pulses and programmable PWM dead-time pulses.

In addition, the advanced timer has break input, inside break circuit realizing write protection function that application program can be protected. Break sources can be break input (BKIN) pin also can be a clock failure events. The clock failure is caused by a reset the clock controller generated by the clock security system. When a break event occurs (the selected levels appear in the break input) will put the timer's output signals in a reset state or in a known state, so protecting motor safety when the motor fails. When Experiment, using SVPWM algorithm to generate 6-channel programmable PWM signals with programmable dead-time and variable output polarity, through the isolated drive unit, sent to intelligent power modules to achieve inverter output. 


\subsection{Isolated Current Sensor Method (ICS)}

To make an accurate implementation of the FOC algorithm, two of three phase stator currents must be read. Which two phase current should be read is determined by current sector of SVPWM. And it is necessary that the implementation of software modules and hardware is connectting consistency and that A/D converters and PWM output signals are synchronized. Current detection hardware architecture is shown in Fig.4, the A, $\mathrm{B}$ phase currents of two bridge inverter arm are respectively detected and sent to the ADC injection channel groups. After stator currents injection (JEOC) conversion FOC algorithm is beginning to be implemented, meanwhile the external ADC trigger is disable until the next count update event of advanced timer generattion.Reading the phase current, The structuret do not need the bridge arm is open. Each time, It do not need to wait for some time when bridge arm switch state has been changed. So it can always use the update event to trigger the ADC, so that the duty cycle has been up to $100 \%$. As the STM32 microcontroller ADC and advanced high-performance timer, you can read at any time PWM cycle current. Of course, we hope that the current sampling point no major changes in different sectors.

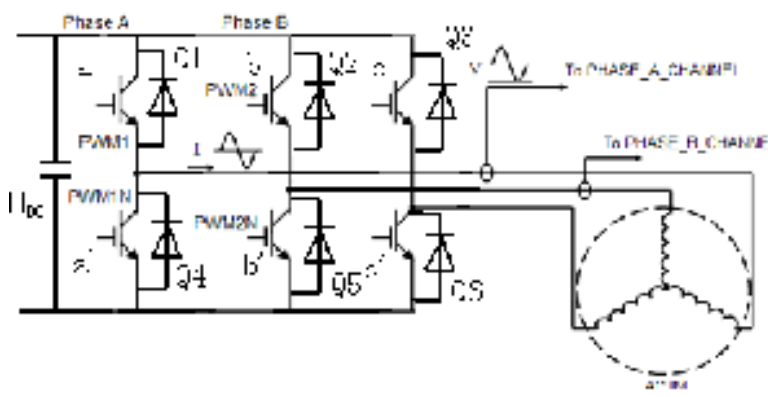

Fig.4 hardware architecture of ICS

\subsection{Serial Communication}

STM32 microcontroller has multiple communication modules, such as UART asynchronous serial. It can be easily achieved communication with computer. The computer mainly implements setting frequency difference control method and motor control parameters - the frequency values given. In addition, The computer will receive and display the speed information transfered from micro-controller.

\subsection{SVPWM algorithm implemention based on FOC}

The technology of space voltage vector pulse width modulation (SVPWM) sees inverter and motor as a whole. From the perspective of motor, it will focus on how to make the motor obtain a circular magnetic field of constant amplitude, namely, sinusoidal magnetic flux, so as to achieve a high control performance. In the variable frequency control experimental system.In Fig.4 the three phase voltage inverter is composed of six power switch devices Qi $(i=0,1 \ldots 6)$ of which the motor phase voltages are composited. And its line voltages are dependent on six power switches state. When a transistor of the upper half part of inverter bridge is opening, that is $\mathrm{a}, \mathrm{b}$ or $\mathrm{c}$ is 1 , while its bottom half part is closed that is $\mathrm{a}^{\prime}, \mathrm{b}^{\prime}$ or $\mathrm{c}^{\prime}$ is 0 . Line voltage vector $\left[U_{a b} U_{b c} U_{c a}\right]^{T}$, phase voltage vector $\left[U_{a} U_{b} U_{c}\right]^{T}$ and the switching variable vector $[a b c]^{T}$ these three relation expressions between each other as Formula (2) and Formula (3) show:

$$
\begin{aligned}
& {\left[\begin{array}{l}
U a \\
U b \\
U c
\end{array}\right]=\frac{1}{3} U_{D C}\left[\begin{array}{ccc}
2 & -1 & -1 \\
-1 & 2 & -1 \\
-1 & -1 & 2
\end{array}\right]\left[\begin{array}{l}
a \\
b \\
c
\end{array}\right]} \\
& {\left[\begin{array}{l}
U a b \\
U b c \\
U c a
\end{array}\right]=\frac{1}{3} U_{D C}\left[\begin{array}{ccc}
1 & -1 & 0 \\
0 & 1 & -1 \\
-1 & -1 & 1
\end{array}\right]\left[\begin{array}{l}
a \\
b \\
c
\end{array}\right]}
\end{aligned}
$$

The $\mathrm{U}_{\mathrm{DC}}$ in formula above-mentioned is the DC voltage of inverter input, or bus voltage.

Switching variable vector $[a b c]{ }^{T}$ has eight different combinatorial values (a, b, c can only be 0 or 1 ), so 
the upper half part of the inverter bridge switch state has a total of eight kinds: U0 [100], U60 [110], U120 [010], U180 [011], U240 [001], U300 [101], O000[000], O111 [111]. It is illustrated that three-phase coordinate system can be transformed into a two-phase coordinate system by coordinate transformation in Formula (4).

$$
\left[\begin{array}{l}
U_{d} \\
U_{q}
\end{array}\right]=\sqrt{\frac{2}{3}}\left[\begin{array}{ccc}
1 & -\frac{1}{2} & -\frac{1}{2} \\
0 & \frac{\sqrt{3}}{2} & -\frac{\sqrt{3}}{2}
\end{array}\right]\left[\begin{array}{l}
U_{A} \\
U_{B} \\
U_{C}
\end{array}\right]
$$

These 8 vectors (including six effective vectors, two zero vectors) composes the basic voltage space vector. The basic principle of SVPWM is to use a number of basic voltage close the given reference of voltage vector space voltage vector Uout, so as to achieve a high control performance.

According to FOC control theory, the stator current is decomposed into direct-axis current $\mathrm{I}_{\mathrm{ds}}$ (magnetic field) and cross-axis current $\mathrm{I}_{\mathrm{qs}}$ (control of torque). $\mathrm{I}_{\mathrm{ds}}$ and $\mathrm{I}_{\mathrm{qs}}$ are then sent into the FOC drive controller. The motor speed is read through the encoder and feedback to the motor given input client (converted to frequency value). The feedback frequency value compares with a given frequency $f^{*}$, constituting the closed-loop speed control of the FOC control. The closed-loop speed regulation control flow chart are given in Fig.5.

FOC control structure is shown in Fig.6. Phase currents $i_{a}, i_{b}$ measured by ICS are transformed into $i_{\alpha}, i_{\beta}$ of $\alpha-\beta$ coordinate system through the Clarke transformation, then transformed into $i_{\mathrm{qs}}$ and $i_{\mathrm{ds}}$ of $d-q$ coordinate system through the Park transformation. And $i_{\mathrm{qs}}$ and $\mathrm{i}_{\mathrm{ds}}$ respectively compare with $\mathrm{i}_{\mathrm{qs}} *$ and $\mathrm{i}_{\mathrm{ds}} *$. While the differences sent into the PID controller, voltage components $V_{\mathrm{qs}}$ and $\mathrm{V}_{\mathrm{ds}}$ of $\mathrm{d}-\mathrm{q}$ coordinate system are obtained and then transformed into the voltage component $V_{\alpha}$ and $V_{\beta}$ of $\alpha-\beta$ coordinate system through reverse Park transformation. And the SVPWM module calculates and assigns the compare register (set the PWM duty cycle) and the prescaler register (set the PWM frequency) of the advanced timer. Thus three phase PWM motor signals are obtained. FOC control algorithm flow chart is given in Fig.7.

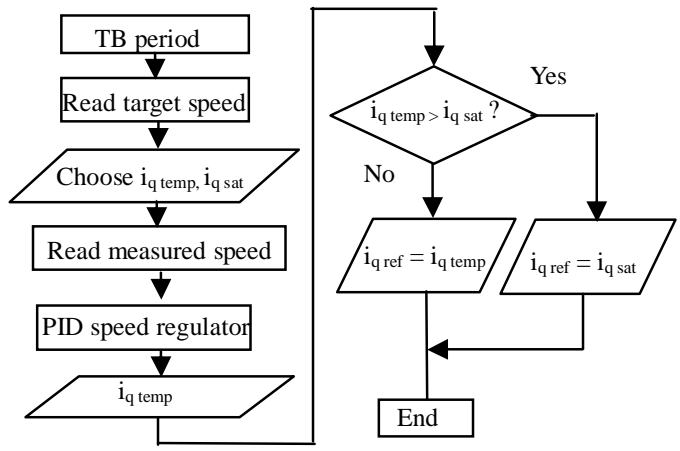

Fig.5 Close-loop speed control flow chart

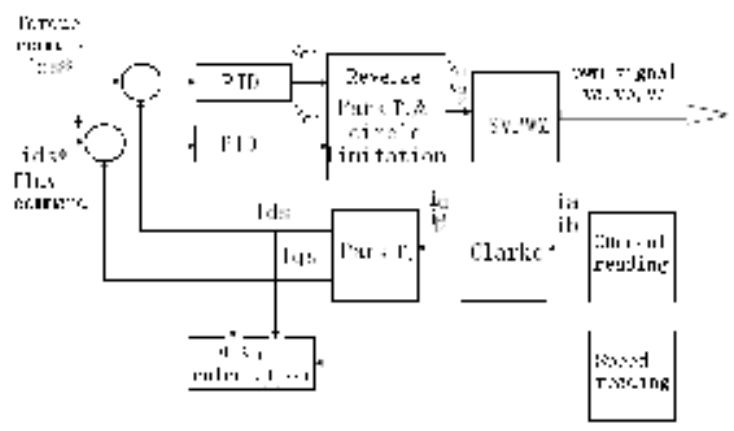

Fig.6 FOC structure 


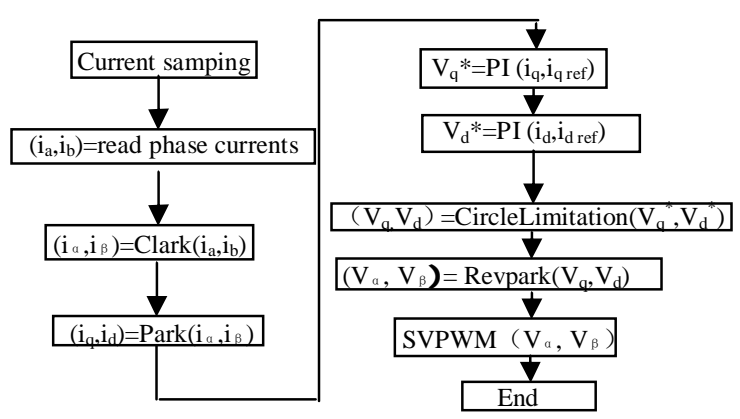

Fig.7 FOC control algorithm flow chart

When the motor is running any of these following three conditions causes the advanced timer to stop exporting PWM signals and the machine to go into fault state. Depending on the source of the fault, an error message is also displayed on the computer screen during Fault state.

(1) Heatsink overtemperature (ADC channel ADC_IN10 and BKIN input).

(2)DC bus over/undervoltage (on ADC channel ADC_IN3).

(3) Overcurrent protection (BKIN input).

\section{Conclusion}

The double-motor structure energy-saving AC variable frequency control experimental system is a new AC speed-regulating experimental system. Theoretical and experimental results show that the system will not only be able to complete many kind of AC motor speed control experiments, but also has good energy saving features and high performances. The experimental system creates a more advanced technical methods and platforms for experiments, development and research. The promotion and appliance of experimental system has significance to improve studing and teaching $\mathrm{AC}$ frequency speed-regulating experiment and theorey.

\section{References:}

[1] Ma Xiaoliang, Decoupling and Regulator Design of Vector Control Systems [J]. Electric drive, 2009, (01).

[2] Chen Zhengshi, Liu Juan, Ye Wei. The Simulation Study of an Energy Saving AC Speed-Adjustment System Based on Regenerate Power Cycle [J]. Electrical and Mechanical Engineering Technology, 2010,320-23.

[3] STM32F10xx Data Sheet, 2008.

[4] STM32F10xx_Library_Manual_ChineseV2.pdf, 2008.

[5] Li Tieying, Zhang Wei, and Chen Zhengshi. Energy-saving AC motors speed-regulating experimental system based on DSP control. Electric Drive Automation, 2007. 